JAMA Internal Medicine | Special Communication | SHARING MEDICINE

\title{
A Research Agenda for Communication Between Health Care Professionals and Patients Living With Serious Illness
}

James A. Tulsky, MD; Mary Catherine Beach, MD, MPH; Phyllis N. Butow, PhD; Susan E. Hickman, PhD; Jennifer W. Mack, MD, MPH; R. Sean Morrison, MD; Richard L. Street Jr, PhD; Rebecca L. Sudore, MD; Douglas B. White, MD, MAS; Kathryn I. Pollak, PhD

IMPORTANCE Poor communication by health care professionals contributes to physical and psychological suffering in patients living with serious illness. Patients may not fully understand their illness, prognosis, and treatment options or may not receive medical care consistent with their goals. Despite considerable research exploring the role of communication in this setting, many questions remain, and a clear agenda for communication research is lacking.

OBSERVATIONS Through a consensus conference and subsequent activities, we reviewed the state of the science, identified key evidence gaps in understanding the impact of communication on patient outcomes, and created an agenda for future research. We considered 7 broad topics: shared decision making, advance care planning, communication training, measuring communication, communication about prognosis, emotion and serious illness communication, and cultural issues. We identified 5 areas in which further research could substantially move the field forward and help enhance patient care: measurement and methodology, including how to determine communication quality; mechanisms of communication, such as identifying the specific clinician behaviors that patients experience as both honest and compassionate, or the role of bias in the clinical encounter; alternative approaches to advance care planning that focus on the quality of serious illness communication and not simply completion of forms; teaching and disseminating communication skills; and approaches, such as economic incentives and other clinician motivators, to change communication behavior.

CONCLUSIONS Our findings highlight the urgent need to improve quality of communication between health care professionals and patients living with serious illness through a broad range of research that covers communication skills, tools, patient education, and models of care.

JAMA Intern Med. 2017;177(9):1361-1366. doi:10.1001/jamainternmed.2017.2005 Published online July 3, 2017.
Editorial page 1256 and Viewpoint page 1237

Author Affiliations: Author affiliations are listed at the end of this article.

Corresponding Author: James A. Tulsky, MD, Department of Psychosocial Oncology and Palliative Care, Dana-Farber Cancer Institute, 450 Brookline Ave, Dana 2016A, Boston, MA 02215-5450 (jamesa_tulsky@dfci.harvard.edu).
$\mathrm{P}$ atients living with serious illness suffer both physically and psychologically. Although many factors contribute, including disease characteristics, quality of care, social determinants, and systems issues, wide consensus exists that poor communication by health care professionals plays a central role. ${ }^{1(p p 117-219)}$ Physical and psychological suffering worsens when patients do not fully understand their illness, prognosis, and treatment options and when clinicians have not sufficiently elicited patients' values. Consequently, patients may receive medical care inconsistent with their goals. ${ }^{2}$

Inadequate communication also leads to higher use of invasive care near death, shorter hospice stays, lower patient quality of life at the end of life, and worse bereavement outcomes for family and caregivers. ${ }^{3}$ Poor-quality communication not only affects patients and families but also contributes to clinician burnout, a troubling outcome given current workforce shortages. ${ }^{4}$ Although considerable research explores the effect of communication on these outcomes, many questions remain. Setting a clear research agenda would move the field forward.

Since the late 1980s, the primary approach to addressing poor communication and unwanted care for patients approaching the end of life has been to promote advance directive completion. Despite legislation encouraging advance directives and numerous dissemination efforts, the outcomes have been disappointing. Initiatives that focus on advance directives alone have changed little in the quality of the experience for seriously patients and families. ${ }^{1}$ Many additional aspects of communication that mediate outcomes in serious illness are insufficiently addressed, including emotion, prognostic awareness, goals of care, spirituality and existential issues, and costs of care. ${ }^{5-8}$ Furthermore, effective interventions have not been disseminated widely into practice. 
In response to these needs, in May 2015, we convened a conference at Duke University School of Medicine, Durham, North Carolina, that brought together the authors of this Special Communication.

The aims of the conference were to (1) review the state of the science in communication research on serious illness, (2) identify the key areas in which investment in research is likely to achieve the greatest returns, and (3) map out an agenda for communication research that includes the identification of potential stakeholders and funding sources. We defined serious illness as life-limiting disease for which most clinicians would not be surprised if the patient died within the year. The majority of research has focused on communication between prescribing clinicians (physicians, nurse practitioners, physician assistants) and their patients, perhaps because they are the clinicians most likely to deliver serious news and have conversations about treatment options toward the end of life. ${ }^{9}$ We also reviewed the empirical work on registered nurse and social worker communication in this setting.

\section{Methods}

We divided the field into 10 broad topic areas, and each expert presented a summary and a focused literature review of their assigned area. After these reviews and discussion, the group summarized what had been learned and created an initial list of research priorities. We used the nominal group technique, a structured iterative ranking process of problem identification, solution generation, and decision making, to develop a final set. ${ }^{10}$ In October 2015, we convened a workshop with additional palliative care and communication experts at the National Palliative Care Research Center Annual Foley Retreat, presented our findings, and received feedback.

\section{Findings}

After the 10 presentations, we organized the broad topic areas under 7 categories: shared decision making, advance care planning, communication training, measuring communication, communication about prognosis, emotion and serious illness communication, and cultural issues.

\section{Shared Decision Making}

Ethical, patient-centered care depends on shared decision making. This has been defined as "a collaborative process that allows patients (or their surrogates) and clinicians to make healthcare decisions together, taking into account the best scientific evidence available, as well as the patient's values, goals, and preferences."11

By what criteria can we judge that shared decision making has been achieved? One perspective argues that evidence of a discussion of medical information that includes patients' values and shared deliberation meets this standard. ${ }^{12,13}$ Alternatively, others posit that shared decision making has occurred only if there is a documented discussion about patient preferences for decision making, such as whether the patient prefers patient-driven, physician-driven, or shared approaches. This documentation allows for a determination of whether the patient's preferences have been followed. ${ }^{14,15}$ Although both approaches have value, communication research may benefit most from a substantive definition that includes specific elements of a conversation.

Given this understanding, does shared decision making occur in practice? Patients and surrogates may have variable role preferences. ${ }^{16}$ Furthermore, studies have shown that physicians frequently fail to discuss options robustly or elicit patients' values in decision-making encounters. ${ }^{17} \mathrm{~A}$ further problem arises when patients with mild cognitive impairment who retain the ability to make decisions about their medical care are excluded. In addition, discordance between patients' desired and actual roles in decision making may be associated with psychological distress. ${ }^{18}$ Finally, consensus is lacking on whether shared decision making should be evaluated on the basis of observed outcomes, such as audio recorded visits or medical record documentation of patient preferences, or patient self-report.

More research is needed to define shared decision making, observe how it unfolds within clinical encounters, and determine how patient attributes affect the shared decision-making process.

\section{Advance Care Planning}

Advance care planning is a process that supports adults at any age or stage of health in understanding and sharing their personal values, life goals, and preferences regarding future medical care. ${ }^{19}$ Although a broad concept that includes many steps, most advance care planning research has focused on tools used to document endof-life treatment preferences, such as advance directives and POLST (Physician Orders for Life Sustaining Treatment) forms. Studies have explored various types of forms and facilitated processes in different age groups, diseases, and the general public. Results of these studies have been mixed. Advance directives are often not available when needed, ${ }^{20}$ and their impact on both costs and care received is unclear. ${ }^{21,22}$ Disparities in literacy and culture compound the problem. Forms are usually written beyond the 12th grade level, only in English, and are oriented toward Western cultural norms about autonomy and decision making. ${ }^{23(p p 160-179)}$

Furthermore, little is known about the communication that occurs between patients, clinicians, and caregivers that leads to the creation of these documents and the clarification of patients' preferences. Despite the completion of forms, patients may lack sufficient rapport with their clinician to discuss their wishes, patients' affect may not be addressed, overall values and life goals may be unexplored, and patients may be poorly informed about their prognosis, medical condition, and choices. ${ }^{1}$

Despite these limitations, some recent efforts have led to more effective advance care planning education, discussion, and completion. Patient videos that clearly describe potential outcomes have affected patient preferences, as have low-literacy forms and websites. ${ }^{24,25}$ Coordinated, comprehensive system-level efforts that enhance measurement and provide patient-facing tools, clinician training, and electronic health record documentation have demonstrated increased advance directive completion and documentation of patients' preferences, better matches between preferences on the directives and treatments received, increased patient and family satisfaction, and lower surrogate anxiety and depression. ${ }^{26,27}$ Greater involvement by social workers and nurse practitioners may lead to greater advance directive completion rates. ${ }^{28}$

For serious and advanced illness, the POLST paradigm is an approach for documenting treatment preferences as medical orders 
that has become a standard component of advance care planning. The National POLST Paradigm Task Force was convened in 2004 to establish quality standards and to assist states in developing POLST paradigm programs. ${ }^{29}$ This oversight body with representatives from each participating state program now endorses programs in 24 states and recognizes 24 others as developing. Although research suggests that POLST orders that reflect patients' choices to limit treatments are associated with lower rates of hospitalization, implementation at the patient level has been variable and the research is not definitive. $^{30}$

The literature on advance care planning leaves many unanswered questions. When should advance care planning be introduced? Who can best facilitate advance care planning, and can patients and their loved ones effectively do some planning on their own? How should advance care planning be documented in the medical record? Which approaches yield the greatest likelihood of goal-concordant care: purely patient-facing interventions, purely clinician-facing interventions, one-on-one facilitated discussions, counseling about advance care planning conducted in small groups of patients, or a combination of approaches tailored to patients' preferences? Finally, which outcomes of advance care planning communication are most important for clinical care?

\section{Communication Training}

There are multiple approaches to effectively train clinicians in serious illness communication skills, including face-to-face workshops, feedback on actual medical encounters both live and audiorecorded, online interactive courses, and innovative computerized simulation approaches. ${ }^{8,31-34}$ These approaches differ from traditional didactic training by ensuring that learners observe examples of high-quality communication, practice the skills, and receive feedback. Although successful in changing clinician behavior, little is known about the long-term effects of this training, the intensity of the interventions that are necessary for success, the value of follow-up skills training, how to engage unmotivated learners, and how to bring such programs to scale.

Several models have been shown, in small studies, to be effective. VitalTalk, of which one of us (J.A.T.) is a founder and director, ${ }^{35}$ has created a structured model of communication skills teaching that allows for greater standardization of training. Hundreds of facilitators, trained in the method, now host their own local courses, and VitalTalk provides them with technical support. Kaiser Permanente has implemented the Four Habits model (not specifically targeted at serious illness), which uses a combination of small group courses and online tools to teach clinicians to adopt 4 habits in every encounter: invest in the beginning, elicit the patient's perspective, demonstrate empathy, and invest in the end. ${ }^{36}$ This intervention has been credited with sustained improvement in patient satisfaction scores. The Serious Illness Communication Project ${ }^{37}$ combines brief communication skills training with a discussion guide and other systems supports. ${ }^{38}$ Respecting Choices offers advance care planning skills training primarily for nurses and social workers. Facilitator certification is provided through standardized courses in combination with systems change principles to promote a culture of person-centered care. ${ }^{27,39}$ Kaiser created its training for the benefit of its own health system; VitalTalk, the Serious Illness Communication Project, and Respecting Choices receive support from philanthropic foundations and also market their services to individuals and health systems. Online approaches have also been shown to be effective in small individual trials ${ }^{8}$; the potential for wider dissemination requires evaluation.

Future directions include disseminating effective interventions through existing mechanisms, such as electronic health records that trigger and guide conversations or maintenance of certification for health professionals that provides an incentive for training in communications about serious illness. Another opportunity is to create quality measures for communication and to pay clinicians who achieve specified communication goals, such as documentation of the conversation, minimal scores on patient surveys, or even quality as determined by audiorecorded encounters. The Centers for Medicare \& Medicaid Services decision in 2016 to pay for advance care planning visits is encouraging. ${ }^{40}$ However, on the basis of prior evidence that advance directives alone do not change patient outcomes, ${ }^{1}$ this effort is likely to increase goal-concordant care only if accompanied by skills training and health system support. Studies of the health system-level impact of communication skills training should be conducted using appropriate research methods, such as cluster randomized trials that account for the possibility of diffusion of an intervention throughout a clinical practice.

\section{Measuring Communication}

To improve the quality of communication, one must be able to measure it. In this domain, measurement includes both what occurs during a conversation (eg, actual words spoken) and how those words are perceived (eg, what patients and clinicians hear). Outcomes of communication include patient trust, satisfaction, decision quality, and health care use. There are several measures for coding actual utterances, including the Verona Coding Definitions of Emotional Sequences (VR-CoDES), ${ }^{41}$ the Roter Interactional Analysis System, ${ }^{42}$ the Street patient participation and physician partnership tool, ${ }^{43}$ and a number of content coding systems for specific behaviors, such as shared decision making. ${ }^{8,44}$ These scientifically validated methods enable investigators to quantitatively and powerfully describe actual clinical interactions, yet their dependence on human manual coding makes them slow and cumbersome and not easily disseminated. For such methods to provide feedback to clinicians or reviewers on a mass scale, high-quality automated coding will be required. Nonverbal communication also affects patient outcomes, yet its measurement is less well developed. ${ }^{45}$

Several challenges remain, however. Of the numerous survey measures about communication quality, it is not known which should be used for what patient populations and in what stage of illness. What is the role of measuring direct communication behavior as an outcome as compared with asking patients to report on communication quality? Moreover, many of these methods of measuring communications have not been fully validated with patient and family perceptions of communication.

\section{Communication About Prognosis}

Most clinicians are reluctant to talk about prognosis. They worry about causing distress and loss of hope. ${ }^{46}$ As a result, some physicians discuss prognosis in vague or overly optimistic terms, avoid the topic unless the patient insists, or focus the discussion on treatment. ${ }^{9}$ Physicians' estimates of prognosis are often overly optimistic as compared with actual outcomes. ${ }^{47,48}$ Patients tend to be even more optimistic than their physicians; nevertheless, they say they want 
prognostic information. ${ }^{49,50}$ Furthermore, with the exception of 1 recent study, ${ }^{51}$ the evidence suggests that prognosis communication does not take away hope, cause depression, increase anxiety, or harm the relationship with the clinician. ${ }^{3,6,7,51,52}$ In contrast, there is some evidence that communication about prognosis may support hope and peace of mind, even when prognosis is poor. ${ }^{5}$

Most data on communication about prognosis is in oncology; less is known about how prognosis is managed in other illnesses with longer and less certain disease trajectories. It is also not known how improving prognostic understanding affects decision making, particularly among patients who prefer not to even discuss the topic.

\section{Emotion and Serious Illness Communication}

Discussing serious illness can be frightening and overwhelming for patients and families. Clinicians worry about taking away patients hope, must manage their own emotions, and often do not possess the tools to address strong emotional reactions during clinical encounters. ${ }^{8}$ Clinicians engaging in conversations about serious illness have 3 primary tasks-establishing connection; eliciting values, goals, and preferences; and delivering information. ${ }^{53}$ Providing information works best when clinicians tailor the amount and type to the individual patient and to the patient's emotional state. Unfortunately, clinicians often share this information with patients who are already upset and may be unable to process what they are hearing. ${ }^{54}$ Allowing patients to express their emotions and to be heard increases their sense of being cared for, conveys relationship, and facilitates understanding and decision making. ${ }^{55}$

It is important to learn how much and what kinds of emotions affect decision making, and how the effect of emotions on decision making varies among patients. How do patient emotions change over time, and how does the quality of the communication influence these changes? And how can physicians facilitate patient emotional expression and involvement?

\section{Cultural Issues}

Cultural issues play an important role in how patients receive information and express emotion. ${ }^{56}$ Research has provided insight into cultural differences in patient and clinician preferences, norms and traditions, and levels of trust in the health care system. ${ }^{57}$ Furthermore, research has highlighted racial, ethnic, and language disparities in quality of care. For example, both African American patients and clinicians have documented preferences for more aggressive care at the end of life compared with their white counterparts. African American patients also are less likely to be enrolled in hospice, to have do not resuscitate orders, ${ }^{58}$ and to use health care professionals as their primary source of advice. ${ }^{59}$ Studies have also found that East Asian families more often have preferences for indirect or no disclosure to the patient directly in the face of serious chronic illness, and greater preferences for family engagement in decision making. ${ }^{60}$

These observations have prompted recommendations for culturally appropriate communication that are not always evidence based, and it is not clear how cultural competency differs from patient-centered communication. ${ }^{61}$ It is not known how clinicians can best communicate with patients with various cultural and individual preferences. Nor is it known which communication strategies work best to assess patients' needs and tailor care to the individual patient within their cultural context.
Priorities for Communication Research in Serious Illness

Based on the review, we identified 5 areas in which further research and improvements in communications could substantially move the field forward and help to enhance patient care.

\section{Measurement and Methodology}

Greater consensus is needed on how to define and measure communication quality and outcomes. Coding systems should be more uniform, and investigators would benefit by knowing which tools are most efficient and serve which research questions best. Communication analysis tools should be more sensitive, more efficient (and thereby scalable), and better able to evaluate the impact of specific clinician words and expressions. There should be more evaluation of nonverbal communication and how it affects outcomes. Communication analysis should be more culturally and socioeconomically appropriate, and studies should include underrepresented populations in instrument development and validation. More research should be conducted in areas of medicine other than oncology. Other measurement needs include greater use of longitudinal assessments, evaluation of how large a "dose" of a communication approach is required to achieve an outcome, clarification of how many conversations are needed to assess an outcome, evaluation of communication with multiple parties (ie, patient, family, clinicians) present, and more multisite trials. Finally, to translate interventions into practice in a timely manner, rapid assessment and pragmatic trials that account for real-life challenges to implementation should be adopted for communication studies.

\section{Mechanisms of Communication}

Work in communication in serious illness is often atheoretical and focuses on blunt interventions without a specific understanding of the impact of discrete communication elements and behaviors. To develop more targeted interventions requires a broader understanding of the "basic science" of communication. Achieving this will require collaboration between clinical and communication science investigators to address questions at a more rudimentary level than are usually studied. Examples of such questions would be, "Is it possible to move patients away from an optimistic bias without causing harm?" "What clinician behaviors do patients experience as both honest and compassionate?" or "How does one promote clinician curiosity, resilience, and presence?" Broad topic areas include teambased communication, clinician communication capacities and motivation, management of bias in the medical encounter, tailoring communication to individual patients, and the effects of social media communication on the experience of serious illness.

\section{Alternative Approaches to Advance Care Planning}

Research on advance care planning and advance directives has not translated into a significant effect on outcomes. Systems approaches, such as POLST, hold promise but evidence is limited. Although documentation is important, future research should focus on the quality of advance care planning communication rather than just the completion of documents. Innovative ways to capture and share patient preferences should account for the trajectory of illness and readiness to engage in advance care planning. A focus should be preparing patients and surrogates for in-themoment decision making, as well as documenting general preferences for care that may not be applicable when specific situations 
arise. Finally, shared decision making in this context may need to be redefined with attention to the role of the family, the impact of emotion, what effective deliberation looks like, and clinician behaviors that enable expression of values and goals.

\section{Teaching and Disseminating Communication Skills}

There is little science about effective teaching methods to promote long-term change. Research should focus on whether feedback is an essential element, how methods can be scaled, and how best to incorporate decision aids into conversations. Furthermore, little is known about communication by and members of the interdisciplinary professional team (including physicians, nurses, social workers, chaplains, therapists), and how to train nonphysicians such as nurses, social workers, or chaplains to conduct specific tasks currently performed primarily by physicians, nurse practitioners, and physician assistants.

\section{Identifying Approaches to Change Communication Behavior}

Educating clinicians, nurses, and social workers is only one approach to improving communications for patients with serious ill- ness. Research should explore the efficacy of multiple approaches that capitalize on other levers in the system. These might include payment approaches that incentivize the time spent on communication, or electronic health record prompts that remind clinicians of serious illness conversations and guide them through the discussion. Alternatively, clinicians may be motivated by sharing their adoption of communication behaviors with others in their practices.

\section{Conclusions}

Improving communication between clinicians and patients living with serious illness can alleviate physical and psychological suffering. There is an urgent need to improve quality of communication in serious illness through a broad range of research that covers communication skills, tools, patient education, and models of care. By focusing research in these identified priority areas, investigators and entities that sponsor research can make substantial strides toward an enhanced patient and clinician experience.

\section{ARTICLE INFORMATION}

Accepted for Publication: April 10, 2017.

Published Online: July 3, 2017. doi:10.1001/jamainternmed.2017.2005

Author Affiliations: Department of Psychosocial Oncology and Palliative Care, Dana-Farber Cancer Institute, Boston, Massachusetts (Tulsky); Division of Palliative Medicine, Brigham and Women's Hospital, Boston, Massachusetts (Tulsky); Department of Medicine, Johns Hopkins School of Medicine, Baltimore, Maryland (Beach); Psycho-Oncology Co-Operative Research Group, Centre for Medical Psychology and Evidence-Based Medicine, School of Psychology, University of Sydney, New South Wales, Australia (Butow); School of Nursing, Indiana University, Indianapolis (Hickman); Department of Pediatric Oncology. Dana-Farber Cancer Institute, Boston, Massachusetts (Mack); Brookdale Department of Geriatrics and Palliative Medicine, Icahn School of Medicine at Mount Sinai, New York, New York (Morrison); Michael E. DeBakey Veterans Affairs Medical Center, Houston, Texas (Street); Baylor College of Medicine, Houston, Texas (Street); Department of Communication, Texas A\&M University, College Station (Street); Division of Geriatrics, Department of Medicine, University of California-San Francisco (Sudore); San Francisco Veterans Affairs Medical Center, San Francisco, California (Sudore); Department of Critical Care Medicine, University of Pittsburgh School of Medicine, Pittsburgh, Pennsylvania (White); Cancer Control and Population Sciences, Duke Cancer Institute, Duke University, Durham, North Carolina (Pollak); Department of Community and Family Medicine, Duke University, Durham, North Carolina (Pollak).

Conflict of Interest Disclosures: Dr Tulsky reports that he is a founder and director of VitalTalk, which is a nonprofit 501(c)(3) organization, and that he receives no compensation for his work with VitalTalk. Dr Hickman reports that she is a member of the National POLST Paradigm Task Force Research Committee and Executive Director of the Indiana Patient Preferences Coalition, as well as organizational faculty for Respecting Choices Last
Steps. She receives no compensation for these activities. No other disclosures are reported.

Funding/Support: We are grateful to the Emily Davie and Joseph S. Kornfeld Foundation for funding this work, and for administrative support from the National Palliative Care Research Center, a nonprofit center that is funded by multiple foundations, committed to stimulating, developing and funding research directed at improving care for seriously ill patients and their families.

Role of the Funder/Sponsor: Neither funding organization had any role in the design and conduct of the work; collection, management, analysis, and interpretation of the data; preparation, review, or approval of the manuscript; and decision to submit the manuscript for publication.

\section{REFERENCES}

1. Institute of Medicine. Dying in America: Improving Quality and Honoring Individual Preferences Near the End of Life. Washington, DC: National Academy of Sciences; 2014.

2. Bernacki RE, Block SD; American College of Physicians High Value Care Task Force. Communication about serious illness care goals: a review and synthesis of best practices. JAMA Intern Med. 2014;174(12):1994-2003.

3. Wright AA, Zhang B, Ray A, et al. Associations between end-of-life discussions, patient mental health, medical care near death, and caregiver bereavement adjustment. JAMA. 2008;300(14): 1665-1673.

4. Thorne SE, Bultz BD, Baile WF, SCRN Communication Team. Is there a cost to poor communication in cancer care? a critical review of the literature. Psychooncology. 2005;14(10):875-884.

5. Mack JW, Wolfe J, Cook EF, Grier HE, Cleary PD, Weeks JC. Hope and prognostic disclosure. J Clin Oncol. 2007;25(35):5636-5642.

6. Mack JW, Wolfe J, Grier HE, Cleary PD, Weeks JC. Communication about prognosis between parents and physicians of children with cancer: parent preferences and the impact of prognostic information. J Clin Oncol. 2006;24(33):5265-5270.
7. Wolfe J, Klar N, Grier HE, et al. Understanding of prognosis among parents of children who died of cancer: impact on treatment goals and integration of palliative care. JAMA. 2000;284(19):2469-2475.

8. Tulsky JA, Arnold RM, Alexander SC, et al. Enhancing communication between oncologists and patients with a computer-based training program: a randomized trial. Ann Intern Med. 2011; 155(9):593-601.

9. The AM, Hak T, Koëter G, van Der Wal G. Collusion in doctor-patient communication about imminent death: an ethnographic study. BMJ. 2000;321(7273):1376-1381.

10. Boddy C. The nominal group technique: an aid to brainstorming ideas in research. Qual Market Res. 2012;15(1):6-18.

11. Kon AA, Davidson JE, Morrison W, Danis M, White DB; American College of Critical Care Medicine; American Thoracic Society. Shared decision making in intensive care units: an American College of Critical Care Medicine and American Thoracic Society policy statement. Crit Care Med. 2016;44(1):188-201.

12. Charles C, Whelan T, Gafni A. What do we mean by partnership in making decisions about treatment? BMJ. 1999;319(7212):780-782.

13. Elwyn $G$, Hutchings $H$, Edwards $A$, et al. The OPTION scale: measuring the extent that clinicians involve patients in decision-making tasks. Health Expect. 2005;8(1):34-42.

14. Kon AA. The shared decision-making continuum. JAMA. 2010;304(8):903-904.

15. Johnson SK, Bautista CA, Hong SY, Weissfeld L, White DB. An empirical study of surrogates' preferred level of control over value-laden life support decisions in intensive care units. Am J Respir Crit Care Med. 2011;183(7):915-921.

16. Hubbard G, Kidd L, Donaghy E. Preferences for involvement in treatment decision making of patients with cancer: a review of the literature. Eur J Oncol Nurs. 2008;12(4):299-318.

17. Tulsky JA, Fischer GS, Rose MR, Arnold RM. Opening the black box: how do physicians 
communicate about advance directives? Ann Intern Med. 1998;129(6):441-449.

18. Gries CJ, Engelberg RA, Kross EK, et al. Predictors of symptoms of posttraumatic stress and depression in family members after patient death in the ICU. Chest. 2010;137(2):280-287.

19. Sudore RL, Lum HD, You JJ, et al. Defining advance care planning for adults: a consensus definition from a multidisciplinary delphi panel. J Pain Symptom Manage. 2017;53(5):821-832.e1.

20. Perkins HS. Controlling death: the false promise of advance directives. Ann Intern Med. 2007;147(1):51-57.

21. Hickman SE, Nelson CA, Perrin NA, Moss AH, Hammes BJ, Tolle SW. A comparison of methods to communicate treatment preferences in nursing facilities: traditional practices versus the physician orders for life-sustaining treatment program. J Am Geriatr Soc. 2010;58(7):1241-1248.

22. Nicholas LH, Langa KM, Iwashyna TJ, Weir DR. Regional variation in the association between advance directives and end-of-life Medicare expenditures. JAMA. 2011;306(13):1447-1453.

23. Institute of Medicine (US) Committee on Understanding and Eliminating Racial and Ethnic Disparities in Health Care. Unequal Treatment: Confronting Racial and Ethnic Disparities in Health Care. Washington, DC: Institute of Medicine; 2003.

24. Volandes $A E$, Paasche-Orlow $M K$, Mitchell SL, et al. Randomized controlled trial of a video decision support tool for cardiopulmonary resuscitation decision making in advanced cancer. J Clin Oncol. 2013;31(3):380-386.

25. Sudore RL, Knight SJ, McMahan RD, et al. A novel website to prepare diverse older adults for decision making and advance care planning: a pilot study. J Pain Symptom Manage. 2014;47(4):674-686.

26. Molloy DW, Guyatt GH, Russo R, et al. Systematic implementation of an advance directive program in nursing homes: a randomized controlled trial. JAMA. 2000;283(11):1437-1444.

27. Detering KM, Hancock AD, Reade MC, Silvester $W$. The impact of advance care planning on end of life care in elderly patients: randomised controlled trial. BMJ. 2010;340:c1345

28. Clark MA, Ott M, Rogers ML, et al. Advance care planning as a shared endeavor: completion of ACP documents in a multidisciplinary cancer program. Psychooncology. 2017;26(1):67-73. 29. National POLST Paradigm Task Force. http://polst.org/. Accessed May 18, 2017.

30. Hickman SE, Keevern E, Hammes BJ. Use of the physician orders for life-sustaining treatment program in the clinical setting: a systematic review of the literature. J Am Geriatr Soc. 2015;63(2):341350.

31. Fallowfield L, Jenkins V, Farewell V, Saul J, Duffy A, Eves R. Efficacy of a Cancer Research UK communication skills training model for oncologists: a randomised controlled trial. Lancet. 2002;359(9307):650-656.

32. Back AL, Arnold RM, Baile WF, et al. Efficacy of communication skills training for giving bad news and discussing transitions to palliative care. Arch Intern Med. 2007;167(5):453-460.

33. Butow P, Cockburn J, Girgis A, et al; CUES Team. Increasing oncologists' skills in eliciting and responding to emotional cues: evaluation of a communication skills training program. Psychooncology. 2008;17(3):209-218.

34. Andrade AD, Bagri A, Zaw K, Roos BA, Ruiz JG. Avatar-mediated training in the delivery of bad news in a virtual world. J Palliat Med. 2010;13(12): 1415-1419.

35. VitalTalk. http://www.vitaltalk.org/. Accessed May 18, 2017.

36. Stein T, Frankel RM, Krupat E. Enhancing clinician communication skills in a large healthcare organization: a longitudinal case study. Patient Educ Couns. 2005:58(1):4-12.

37. Ariadne Labs. Serious Illness Care. https://www.ariadnelabs.org/areas-of-work /serious-illness-care/. Accessed May 18, 2017.

38. Bernacki R, Hutchings $M$, Vick J, et al. Development of the Serious Illness Care Program: a randomised controlled trial of a palliative care communication intervention. BMJ Open. 2015;5 (10):e009032

39. Respecting Choices. http://www .gundersenhealth.org/respecting-choices/. Accessed May 18, 2017.

40. Center for Medicare \& Medicaid Services. Advance Care Planning. https://www.cms.gov /Outreach-and-Education/Medicare-Learning -Network-MLN/MLNProducts/Downloads /AdvanceCarePlanning.pdf. Accessed May 18, 2017.

41. Del Piccolo L, de Haes $H$, Heaven $C$, et al. Development of the Verona coding definitions of emotional sequences to code health providers' responses (VR-CoDES-P) to patient cues and concerns. Patient Educ Couns. 2011;82(2):149-155.

42. Roter $D$, Larson $S$. The Roter interaction analysis system (RIAS): utility and flexibility for analysis of medical interactions. Patient Educ Couns. 2002;46(4):243-251.

43. Street RL Jr, Millay B. Analyzing patient participation in medical encounters. Health Commun. 2001;13(1):61-73.

44. Braddock CH III, Fihn SD, Levinson W, Jonsen AR, Pearlman RA. How doctors and patients discuss routine clinical decisions: informed decision making in the outpatient setting. J Gen Intern Med. 1997;12 (6):339-345

45. Henry SG, Fuhrel-Forbis A, Rogers MA, Eggly S. Association between nonverbal communication during clinical interactions and outcomes: a systematic review and meta-analysis. Patient Educ Couns. 2012;86(3):297-315.

46. Daugherty CK, Hlubocky FJ. What are terminally ill cancer patients told about their expected deaths? a study of cancer physicians' self-reports of prognosis disclosure. J Clin Oncol. 2008;26(36):5988-5993.

47. Christakis NA, Lamont EB. Extent and determinants of error in doctors' prognoses in terminally ill patients: prospective cohort study. BMJ. 2000;320(7233):469-472.
48. Lee SJ, Fairclough D, Antin JH, Weeks JC. Discrepancies between patient and physician estimates for the success of stem cell transplantation. JAMA. 2001;285(8):1034-1038.

49. Hagerty RG, Butow PN, Ellis PM, et al. Communicating with realism and hope: incurable cancer patients' views on the disclosure of prognosis. J Clin Oncol. 2005;23(6):1278-1288.

50. Gramling R, Fiscella K, Xing G, et al. Determinants of patient-oncologist prognostic discordance in advanced cancer. JAMA Oncol. 2016; 2(11):1421-1426.

51. Carson SS, Cox CE, Wallenstein S, et al. Effect of palliative care-led meetings for families of patients with chronic critical illness: a randomized clinical trial. JAMA. 2016;316(1):51-62.

52. Enzinger $A C$, Zhang $B$, Schrag $D$, Prigerson $H G$. Outcomes of prognostic disclosure: associations with prognostic understanding, distress, and relationship with physician among patients with advanced cancer. J Clin Oncol. 2015;33(32):38093816.

53. Back AL, Arnold RM. "Yes it's sad, but what should I do?" moving from empathy to action in discussing goals of care. J Palliat Med. 2014;17(2): 141-144.

54. Siminoff LA, Step MM. A comprehensive observational coding scheme for analyzing instrumental, affective, and relational communication in health care contexts. $J$ Health Commun. 2011:16(2):178-197.

55. Fogarty LA, Curbow BA, Wingard JR, McDonnell K, Somerfield MR. Can 40 seconds of compassion reduce patient anxiety? J Clin Oncol. 1999;17(1):371-379.

56. Butow PN, Sze M, Eisenbruch M, et al. Should culture affect practice? a comparison of prognostic discussions in consultations with immigrant versus native-born cancer patients. Patient Educ Couns. 2013:92(2):246-252.

57. Mystakidou K, Parpa E, Tsilila E, Katsouda E, Vlahos L. Cancer information disclosure in different cultural contexts. Support Care Cancer. 2004;12 (3): 147-154.

58. Johnson KS, Kuchibhatla M, Tulsky JA. What explains racial differences in the use of advance directives and attitudes toward hospice care? J Am Geriatr Soc. 2008;56(10):1953-1958.

59. Trevino KM, Zhang B, Shen MJ, Prigerson HG. Accuracy of advanced cancer patients' life expectancy estimates: the role of race and source of life expectancy information. Cancer. 2016;122 (12):1905-1912.

60. Wuensch A, Tang L, Goelz T, et al. Breaking bad news in China-the dilemma of patients' autonomy and traditional norms: a first communication skills training for Chinese oncologists and caretakers. Psychooncology. 2013;22(5):1192-1195.

61. Saha S, Beach MC, Cooper LA. Patient centeredness, cultural competence and healthcare quality. J Nat/ Med Assoc. 2008;100(11):1275-1285. 